\title{
Modified Aggregation Tree based Algorithm for Load Balancing in MANET
}

\section{Hemalatha, S. Mohanapriya}

\begin{abstract}
Proposed a Load Balancing in Data Aggregation Tree (DAT), to diminish the transmission cost by diminishing the bounce remove a bundle will travel and by combining information into reasonable transitional hubs. For sending information from a hub at level $l$, DAT calculation wisely chooses a hub at level l-1 by utilizing the aggregation proportion related with each sensor hub. We have led broad reproductions for breaking down the exhibition of DAT and contrast the outcome and existing Tree-Adapting calculation. Our tests demonstrate that DAT shows better execution when contrasted with Tree-Adapting calculation in diminishing the absolute vitality cost because of bundle transmissions.
\end{abstract}

Keywords--- Load Balancing, Aggregation, MANET, Traffic, Overload.

\section{INTRODUCTION}

In a flexible framework, move center course of action means describing the direction for the hand-off center with the ultimate objective that it by and large remains at the perfect position in regard to the spots of the centers in its organization set.

An insignificant technique to achieve this could be to have the customary center points send territory updates to the exchange center point at each time minute all through the length of an age. The exchange center point would then consistently enroll its optimal region encourages reliant on these zone updates and position itself as necessities be. Regardless of the way that this procedure would result in a perfect direction for the hand-off center, it is clearly unrealistic in light of the correspondence overheads included.

To address this issue, the proposed system uses adaptability desire figurings to assess the advancement instances of the versatile centers. At the point when the exchange center point concludes its organization set, it forms its optimal position orchestrates at different time minutes over the term of an age using the foreseen territory of the source center points contrasting with the streams in its organization set.

In light of these anticipated position arranges, the handoff center point at that point describes its bearing for the length of the age.

The heading is just the locus of the centers portrayed the game plans at different events during a time using the anticipated position encourages. Evidently, the precision of the desire count nearby the amount of figures in the midst of an age will essentially influence the prepared places of the exchange center.

At the point when the hand-off center portrayed its course for an age, it needs to pass on this information to all of the center points in its organization set. With this effect, the exchange center instructs the customary centers of its determined position organizes at a fixed number of time minutes over the range of an age.

The customary center points would accordingly utilize this information to predict their partition to the hand-off center in order to appropriately set the transmit control when talking with the hand-off center. Most of the estimate plans proposed in the MANET writing rely upon a central model that portrays the flexibility of the center points in the framework.

For example, in the makers anticipate the situation of the center points expecting the centers seek after an unpredictable walk compactness appear. While such computations may result in strong depictions, they most likely won't work honorably if suppositions identifying with the fundamental convey ability show don't hold incredible.

MANET applications frequently require participation among an enormous number of hubs. One model is to ceaselessly screen a zone and report occasions. Another model is a hub conveys a question about intrigued information to various hubs. Along these lines numerous individual information should be gathered and removed to shape some higher-level data. The system of information accumulation and extraction is called information aggregation.

To perform information aggregation in MANET numerous issues must be settled: like constrained power, unsteady system topology, and so forth. The correspondence cost is the most serious issue for information aggregation, since remote connection is an open media with constrained transmission capacity.

Sending huge number of information presents the system much correspondence traffic load. Moreover, much correspondence will deplete the battery intensity of sensors rapidly. In this way how to diminish the correspondence cost must be a significant factor of the aggregation convention.

\footnotetext{
Manuscript received September 16, 2019.

M. Hemalatha, Asst. Professor, Dept of Computer Application, Hindusthan College of Arts \& Science, Coimbatore, T.N, India.

Dr.S. Mohanapriya, Head, Dept of Computer Science, K.S.R College of Arts \& Science for Women, Tiruchengode, T.N, India.
} 


\section{LITERATURE SURVEY}

\begin{tabular}{|c|c|c|}
\hline $\begin{array}{l}\text { Jeungeun Song, Yiming } \\
\text { Miao, Enmin Song, M. } \\
\text { ShamimHossain and } \\
\text { Mohammed F. Alhamid }\end{array}$ & $\begin{array}{l}\text { Propose the RCR-based ideal transfer task and helpful } \\
\text { information delivery (RCR-delivery) plan to give a low- } \\
\text { correspondence overhead information transmission and an } \\
\text { ideal obligation cycle for a given number of agreeable } \\
\text { hubs when the system is dynamic, which empowers some } \\
\text { portion of agreeable hubs to switch into inert status for } \\
\text { further vitality sparing. }\end{array}$ & $\begin{array}{l}\text { The proposed plan fundamentally beats the } \\
\text { current geographic steering plans and } \\
\text { beaconless geographic routings in remote } \\
\text { sensor systems with a profoundly powerful } \\
\text { remote channel and controls vitality utilization, } \\
\text { while ETE unwavering quality is adequately } \\
\text { ensured. }\end{array}$ \\
\hline $\begin{array}{lll}\text { Hongbin } & & \text { Chen } \\
\text { QianZeng and } & \text { Feng } \\
\text { Zhao } & & \end{array}$ & $\begin{array}{l}\text { A proficient sleep scheduling calculation is advanced to } \\
\text { handle the above issue in vitality harvesting sensor } \\
\text { systems. At first, we adjust the probability based } \\
\text { prediction and sleep scheduling (PPSS) calculation to } \\
\text { follow the objective and further utilize another sleep } \\
\text { scheduling calculation we proposed to wake following } \\
\text { hubs when the objective is probably going to be missed } \\
\text { (i.e., it is fruitless to wake next-minute following hubs). } \\
\text { Also, a twofold capacity vitality harvesting architecture is } \\
\text { utilized to build leftover vitality of sensor hubs and to } \\
\text { broaden arrange lifetime. }\end{array}$ & $\begin{array}{l}\text { The proposed sleep scheduling calculation can } \\
\text { improve following execution and drag out } \\
\text { system lifetime contrasted and the PPSS } \\
\text { calculation and the proposed calculation } \\
\text { without vitality harvesting. }\end{array}$ \\
\hline $\begin{array}{l}\text { Jue Hong, Zhuo Li, } \\
\text { Dianjie Lu and Sanglu } \\
\text { Lu }\end{array}$ & $\begin{array}{l}\text { Propose SALB, a sleeping timetable mindful neighborhood } \\
\text { broadcast calculation. In SALB, a commonplace } \\
\text { neighborhood calculation for building associated } \\
\text { overwhelming set is utilized to shape the broadcast spine. } \\
\text { To ensure appropriate transmission of broadcast messages, } \\
\text { a sleep-mindful sending instrument is actualized. Also, } \\
\text { heuristic systems are utilized to diminish the quantity of } \\
\text { transmissions and the broadcast latency. }\end{array}$ & $\begin{array}{l}\text { Adjusted an old style nearby calculation for } \\
\text { building associated commanding set to shape } \\
\text { the broadcast spine and structured a sending } \\
\text { system to handle the occasionally sleeping } \\
\text { issue of hubs. }\end{array}$ \\
\hline $\begin{array}{l}\text { Sha Liu, Kai-Wei Fan, } \\
\text { PrasunSinha }\end{array}$ & $\begin{array}{l}\text { Current sleep-scheduling approaches for sensor organizes } \\
\text { that address vitality utilization either possibly spare vitality } \\
\text { when lingering or have high latencies and low channel use. } \\
\text { Our commitment is a vitality productive sleep scheduling } \\
\text { convention called BSMac for sensor systems while } \\
\text { keeping up high throughput and low latency. BSMac is } \\
\text { based on another architecture called BoostNet in which the } \\
\text { base station broadcasts basic scheduling coordination data } \\
\text { utilizing enormous transmission range to achieve all sensor } \\
\text { hubs in a single jump. }\end{array}$ & $\begin{array}{l}\text { Hubs allots hues successively to the active } \\
\text { connections along the traffic way, and hubs not } \\
\text { on the information way work at a low } \\
\text { obligation cycle. To suit diverse traffic designs, } \\
\text { the quantity of hues is basic. To accomplish } \\
\text { ideal throughput, the base station explores the } \\
\text { system with various most extreme number of } \\
\text { hues intermittently. }\end{array}$ \\
\hline $\begin{array}{l}\text { Niranjan Kumar Ray } \\
\text { and Ashok Kumar } \\
\text { Turuk }\end{array}$ & $\begin{array}{l}\text { Proposed a vitality protection method called Location } \\
\text { Based Topology Control with Sleep Scheduling for } \\
\text { specially appointed systems. It utilizes the element of both } \\
\text { topology control approach and power the board approach. } \\
\text { Like the topology control approach, it endeavors to lessen } \\
\text { the transmission intensity of a hub, which is resolved from } \\
\text { its neighborhood area data. A hub rests state based on the } \\
\text { traffic condition as that of intensity the executives } \\
\text { approach. }\end{array}$ & $\begin{array}{l}\text { A hub rests state just when its nonappearance } \\
\text { does not make nearby parcel in its } \\
\text { neighborhood. We preformed broad recreation } \\
\text { to contrast the proposed plan and existing ones. } \\
\text { Reproduction results demonstrate that the } \\
\text { vitality utilization is lower with increment in } \\
\text { the system lifetime and higher throughput in } \\
\text { the proposed plan. }\end{array}$ \\
\hline
\end{tabular}

\section{PROPOSED WORK}

\subsection{Data Aggregation Tree}

Various components called aggregation calculations are proposed so as to exclude the excess information. Aggregation calculations, subsequent to getting information from a few sensors, process information and preclude the repetition and send the aftereffect of aggregation to the sink. Because of the decrease in information volume, these calculations decline the vitality utilization. Thusly the systems which perform aggregation have more life time and draw more consideration. Notwithstanding referenced upgrades, aggregation diminishes impact and retransmission delay. Information aggregation is performed during directing in remote sensor systems. Finding the course from a few hubs to the sink in a manner that expands the common way and repetition expelling is one of the fundamental targets in these conventions. In aggregation calculations, we should build aggregation traversing tree. The traversing tree is a tree which contains all system hubs and doesn't have any circle. Aggregation component fills in as pursue: every hub detects information from the earth and gets other hub's information, at that point totals these information, in view of the aggregation work and transmits the aggregation result to the sink.

Algorithm. Aggregation tree constructing algorithm Input: $\mathrm{G}=(\mathrm{V}, \mathrm{E})$, radius $\mathrm{r}$;

Output: Data aggregation tree DAT $=(\mathrm{V}, \mathrm{E})$

1. Assuming $\mathrm{V}_{\mathrm{s}}$ as root and $\mathrm{r}$ as radius, construct a breadthfirst searching tree $\mathrm{G}$; 
2. For $v_{\mathrm{I}} \in \mathrm{V}$, let $\mathrm{C}(\mathrm{i})=$ White, Mark (i) -0

3. Let $\mathrm{C}_{\mathrm{vs}}(\mathrm{i})=\mathrm{Black}$, broadcast $\mathrm{Msg}(\mathrm{B})$;

4. For each node $\mathrm{V}_{\mathrm{j}}$ that has received $\mathrm{msg}(\mathrm{B})$;

5. If $\mathrm{C}(\mathrm{j})=$ White, then

6. $\quad$ let $\mathrm{C}(\mathrm{j})=$ Grey and broadcasr Msg $(\mathrm{G})$;

7. If $\mathrm{C}(\mathrm{j})=$ White and " $\mathrm{v}_{\mathrm{i}}\left\|\mathrm{v}_{\mathrm{i}}-\mathrm{v}_{\mathrm{j}}\right\| \leq \mathrm{r}$,

$$
\mathrm{R}_{\mathrm{j}}<\mathrm{R}_{\mathrm{i}}
$$

8. $\quad$ Let $\mathrm{C}(\mathrm{j})=$ Black and broadcast Msg $(\mathrm{B})$;

9. Let $\mathrm{DS}=\left\{\mathrm{v}_{\mathrm{i}} \mid \mathrm{C}(\mathrm{j})=\right.$ Black $\}$;

10. For root $\mathrm{v}_{\mathrm{s}}$, broadcast $\mathrm{Msg}\left(\mathrm{G}_{\text {join }}\right)$;

11. For each node $v_{I}$ that has received $M s g\left(G_{\text {join }}\right)$

12. If $\mathrm{v}_{\mathrm{i}} \in \mathrm{DS}, \operatorname{Mark}(\mathrm{i})=0, \mathrm{C}(\mathrm{i}) \_$Grey, then

13. Let DS $=$ DS U $\left\{\mathrm{v}_{\mathrm{i}}\right\}$, broadcast Msg $(\mathrm{B}$

join), Mark )i) = 1 ;

14. If $v_{i} \in D S, \operatorname{Mark}(j)=0, C(j)+B$ lack, then
15.

Let DS $=\mathrm{DS} U\left\{\mathrm{v}_{\mathrm{i}}\right\}$, broadcast Msg $(\mathrm{G}$

join)

16. Assuming Ds as non-leaf nodes and other nodes as leaf nodes, construct a tree;

17. For all non-root nodes $v_{i} \in V$, let its degree be Deg )i), $\mathrm{H}(\mathrm{i})=$ hops $\left(\mathrm{v}_{\mathrm{i}}, \mathrm{v}_{\mathrm{s}}\right)$;

18. Solve the following optimal problem:

$$
\begin{aligned}
& \mathrm{P}_{\mathrm{i}}=\operatorname{argmin}(\operatorname{Deg}(\mathrm{j})) \\
& \text { s.t }\left\|\mathrm{v}_{\mathrm{i}}-\mathrm{v}_{\mathrm{j}}\right\| \leq \mathrm{t} \\
& \mathrm{H}(\mathrm{i})-\mathrm{H}(\mathrm{j})=1
\end{aligned}
$$

19. Return DAT $=(\mathrm{V}, \mathrm{E})$;

\section{EXPERIMENTAL RESULTS}

Fault Tolerance

Table 1: Comparison table of fault tolerance

\begin{tabular}{|l|l|l|l|}
\hline $\begin{array}{l}\text { Energy Efficient } \\
\text { Neighbor Coverage } \\
\text { Protocol(EENCP) }\end{array}$ & $\begin{array}{l}\text { Secure Enhanced Adaptive } \\
\text { Acknowledgement(SEAACK) }\end{array}$ & $\begin{array}{l}\text { Probability Based Prediction and Sleep } \\
\text { Scheduling(PPSS) }\end{array}$ & $\begin{array}{l}\text { Propos } \\
\text { ed }\end{array}$ \\
\hline $\mathbf{0 . 7 8}$ & 0.42 & 0.54 & 0.18 \\
\hline $\mathbf{0 . 9}$ & 0.94 & 0.77 & 0.38 \\
\hline $\mathbf{1 . 1 1}$ & 1.74 & 0.99 & 0.73 \\
\hline $\mathbf{1 . 4}$ & 2.72 & 1.36 & 1 \\
\hline $\mathbf{1 . 8 9}$ & 2.98 & 1.6 & 1.04 \\
\hline
\end{tabular}

Examination table of adaptation to internal failure depicts three existing techniques energy efficient neighbor coverage protocol (EENCP), Secure Enhanced Adaptive Acknowledgement (SEAACK), Probability Based
Prediction and Sleep Scheduling (PPSS) and one proposed strategy. Contrasted with existing strategies the proposed techniques esteems are low. Proposed strategy esteems begins from 0.18 to 1.04 .

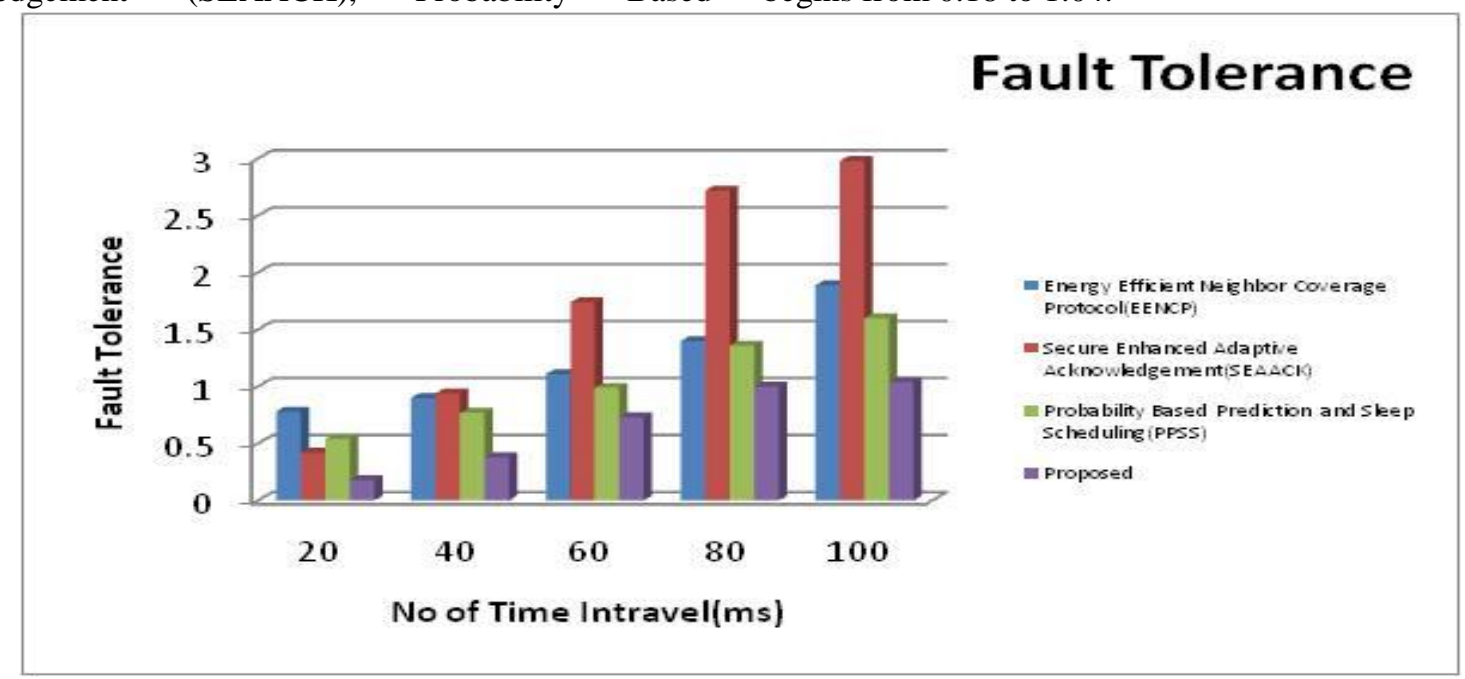

Figure 2: Comparison chart of fault tolerance

The Comparison outline of adaptation to internal failure than other. Proposed method values begins from 0.18 to demonstrates the different values of existing methods and 1.04. proposed method. No of records in x pivot and succession Distribution of node residual energy level in Y hub. At the point when analyzed existing method and proposed method the proposed method values are lower

Table 2: Comparison table of Distribution of node residual energy

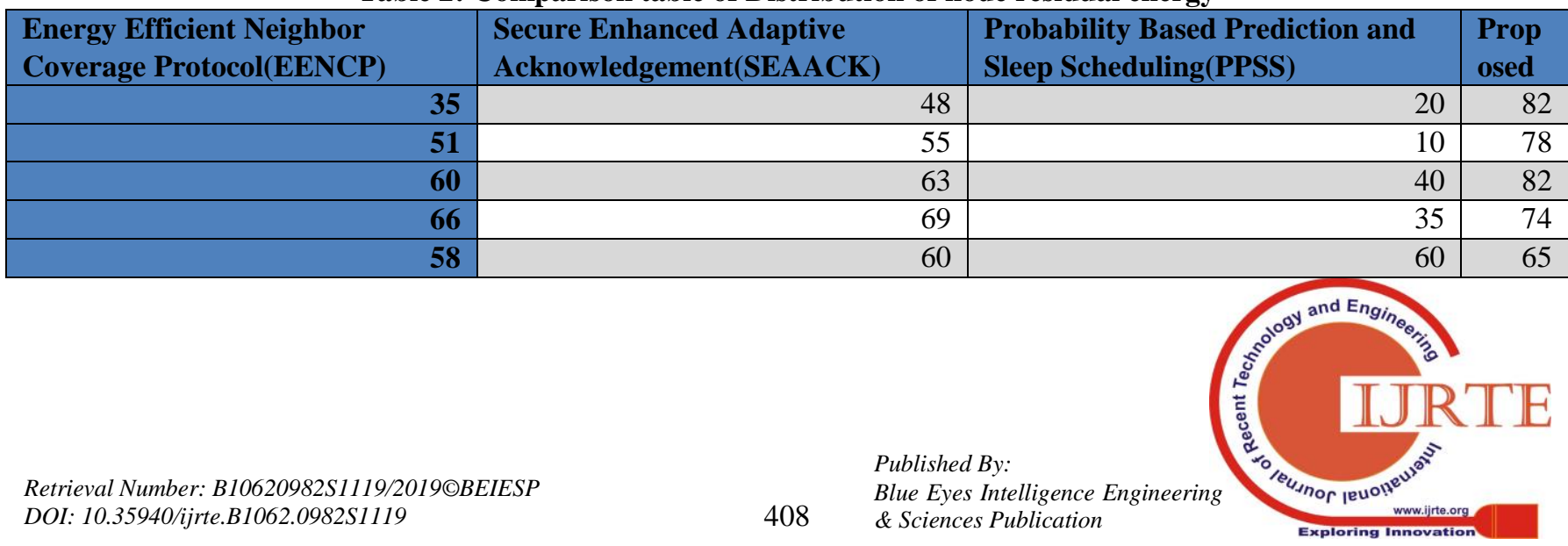


Comparison table of Distribution of node residual energy describes three existing methods Energy Efficient Neighbor Coverage Protocol (EENCP), Secure Enhanced Adaptive Acknowledgements(SEAACK), Probability Based
Prediction and Sleep Scheduling(PPSS) and one proposed method. Compared to proposed method the existing methods values are low. Proposed method values are starts from 82 to 65 .

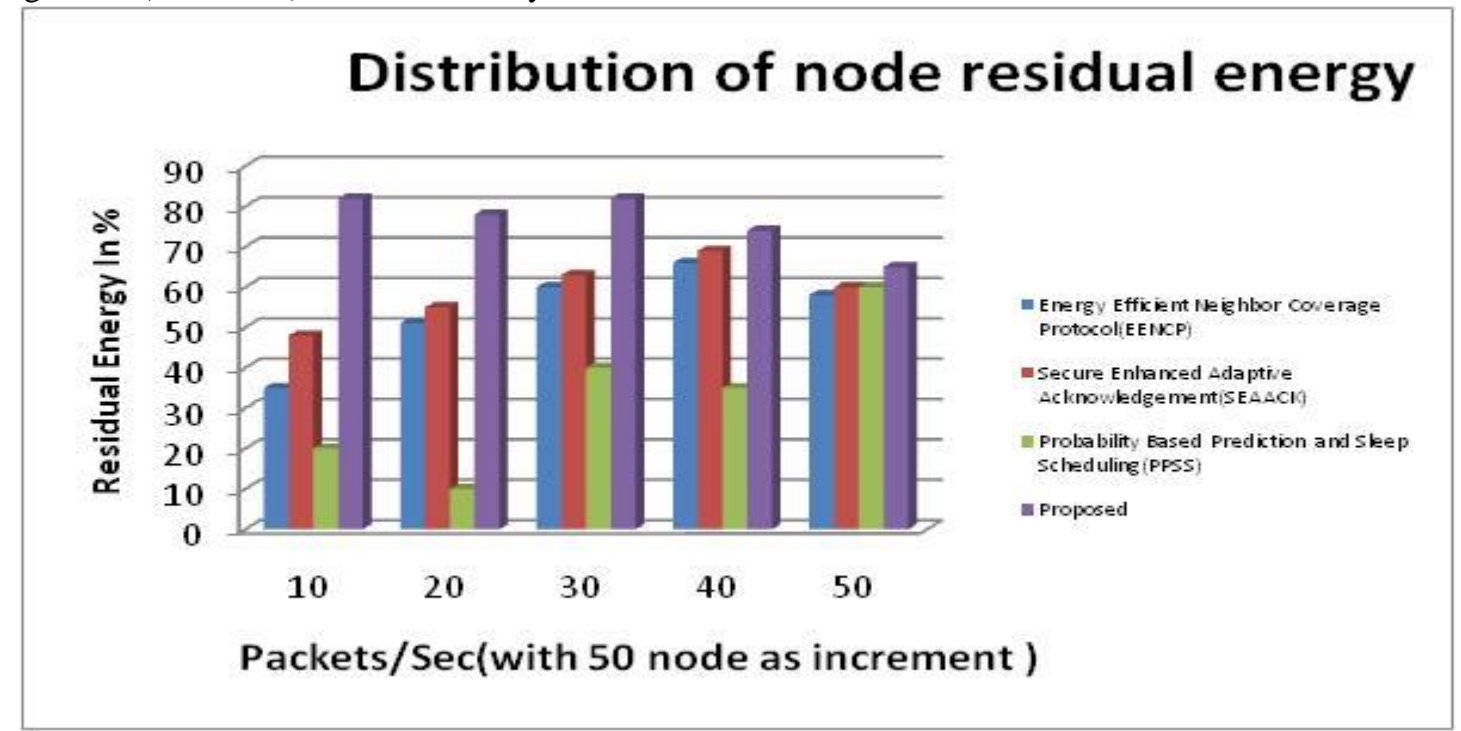

Figure 2: Comparison chart of Distribution of node residual energy

The Comparison chart of Distribution of node residual energy shows the different values of existing methods and proposed method. Packets/Sec (with 50 node as increment) in $\mathrm{X}$ axis and Residual Energy In\% in $\mathrm{Y}$ axis. When compared existing method and proposed method the proposed method values are higher than other. Proposed values are starts from 82 to 65 .

Availability

Table 3: Comparison table of availability

\begin{tabular}{|l|l|l|}
\hline $\begin{array}{l}\text { Energy Efficient Neighbor Coverage } \\
\text { Protocol(EENCP) }\end{array}$ & $\begin{array}{l}\text { Secure Enhanced Adaptive } \\
\text { Acknowledgement(SEAACK) }\end{array}$ & $\begin{array}{l}\text { Propos } \\
\text { Scheduling(PPSS) }\end{array}$ \\
\hline $\mathbf{0 . 1 4}$ & 0.9 & 0.9 \\
\hline $\mathbf{0 . 3}$ & 0.2 & 0.2 \\
\hline $\mathbf{0 . 4}$ & 0.3 & 0.32 \\
\hline $\mathbf{0 . 5 6}$ & 0.37 & 0.39 \\
\hline $\mathbf{0 . 7 7}$ & 0.49 & 0.47 \\
\hline
\end{tabular}

Comparison table of availability describes three existing methods energy efficient neighbor coverage protocol(EENCP), Secure Enhanced Adaptive Acknowledgement(SEAACK), Probability Based Prediction

and Sleep Scheduling(PPSS) and one proposed method Compared to existing methods the proposed methods values are high. Proposed method values starts from 0.22 to 1.1 .

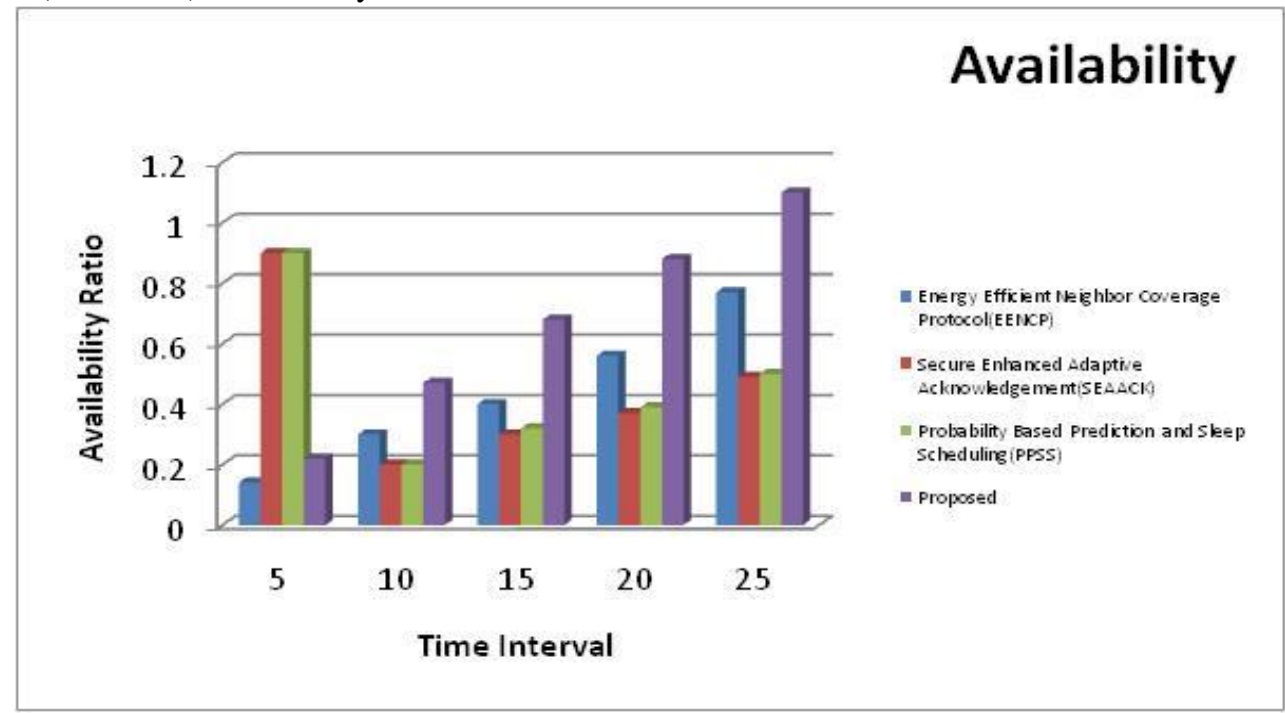

Figure 3: Comparison chart of availability 
The Comparison chart of availability shows the different values of existing methods and proposed method. No of records in $\mathrm{x}$ axis and sequence level in $\mathrm{Y}$ axis. When compared existing method and proposed method the proposed method values are higher than other. Proposed method values starts from 0.22 to 1.1 .

Network Lifetime

Table 4: Comparison table of Network lifetime

\begin{tabular}{|l|l|l|l|}
\hline $\begin{array}{l}\text { Energy Efficient Neighbor } \\
\text { Coverage Protocol(EENCP) }\end{array}$ & $\begin{array}{l}\text { Secure Enhanced Adaptive } \\
\text { Acknowledgement(SEAACK) }\end{array}$ & $\begin{array}{l}\text { Probability Based Prediction and } \\
\text { Sleep Scheduling(PPSS) }\end{array}$ & $\begin{array}{l}\text { Prop } \\
\text { osed }\end{array}$ \\
\hline $\mathbf{1 2 5}$ & 250 & 200 & 345 \\
\hline $\mathbf{1 4 5}$ & 200 & 150 & 310 \\
\hline $\mathbf{9 9}$ & 240 & 100 & 280 \\
\hline $\mathbf{1 6 6}$ & 190 & 180 & 220 \\
\hline $\mathbf{1 8 0}$ & 250 & 196 & 315 \\
\hline
\end{tabular}

This comparison table describes the network lifetime for various packet inter arrival times of three existing methods EENCP, SEAACK, PPSS and proposed method. Comparing these four methods we assume that the packets of proposed

method shows the maximum inter arrival times of to minimal inter arrival times from 345 to 315 . The existing method shows a minimum arrival times than proposed method.

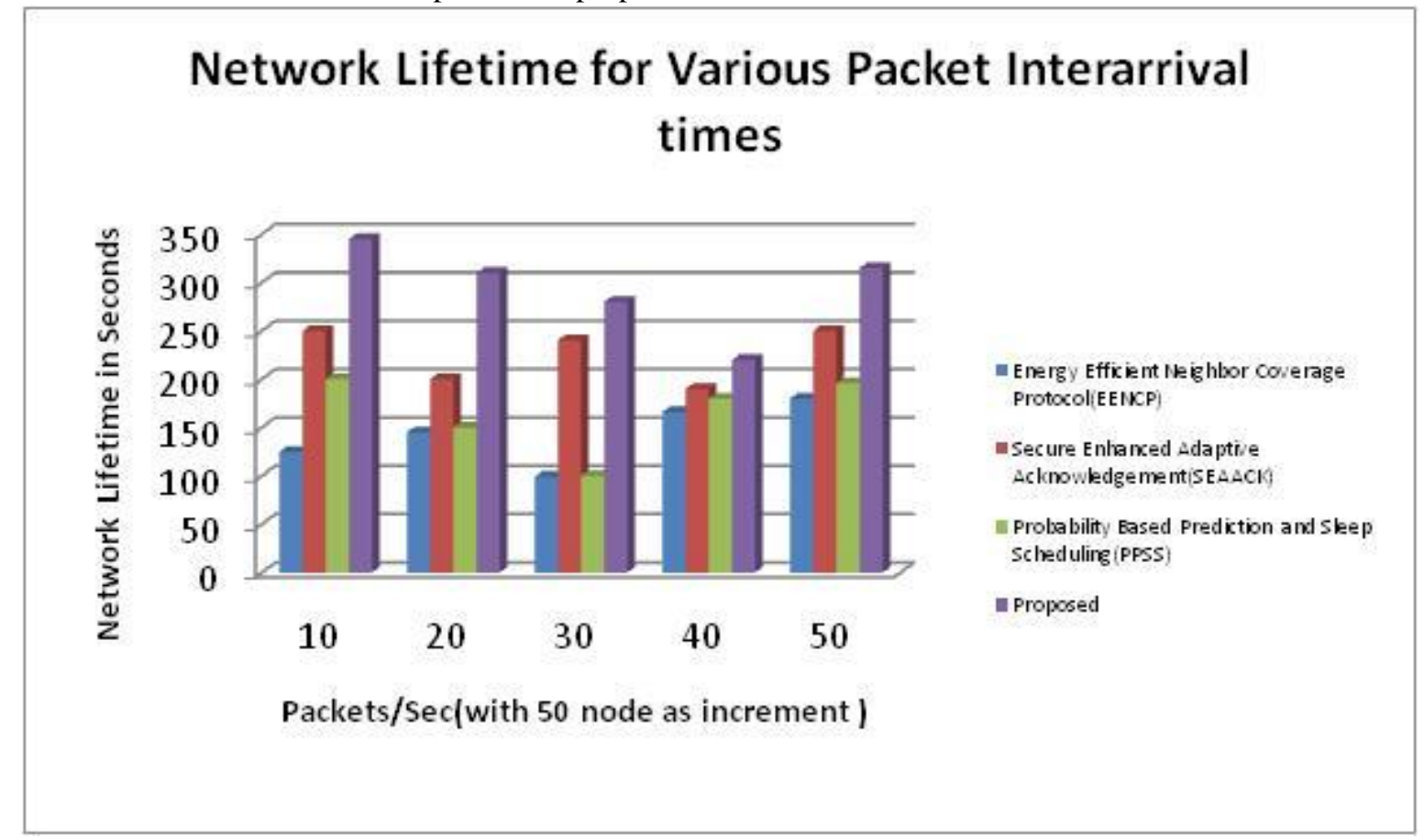

Figure 4: Comparison table of Network lifetime

Comparison chart explains about the network lifetime for various packets inter arrival times of three existing Methods and one proposed method. The comparison of these four methods is been explained using the number of packets in $\mathrm{X}$ axis and the seconds of network lifetime value in $\mathrm{Y}$ - axis. The level of proposed method shows the maximum to minimum value 345 to 315 when compared to existing methods.

False Positive

Table 5: Comparison table of False positive ratio

\begin{tabular}{|l|l|l|l|}
\hline $\begin{array}{l}\text { Energy Efficient Neighbor } \\
\text { Coverage Protocol(EENCP) }\end{array}$ & $\begin{array}{l}\text { Secure Enhanced Adaptive } \\
\text { Acknowledgement(SEAACK) }\end{array}$ & $\begin{array}{l}\text { Probability Based Prediction and } \\
\text { Sleep Scheduling(PPSS) }\end{array}$ & $\begin{array}{l}\text { Prop } \\
\text { osed }\end{array}$ \\
\hline $\mathbf{7 1 0}$ & 600 & 1000 & 500 \\
\hline $\mathbf{7 9 0}$ & 650 & 1290 & 599 \\
\hline $\mathbf{8 3 0}$ & 780 & 1450 & 810 \\
\hline $\mathbf{1 1 0 0}$ & 800 & 1610 & 1000 \\
\hline $\mathbf{6 0 0}$ & 400 & 1700 & 499 \\
\hline
\end{tabular}

This table describes the comparison of false positive ration of three existing method that is EENCP, SEAACK, PPSS method and proposed method. Comparing these four

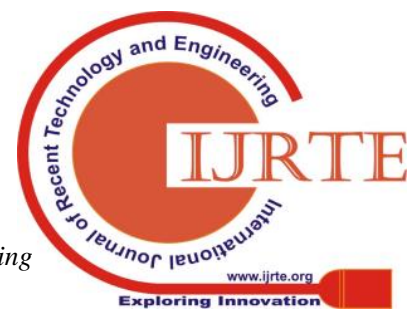




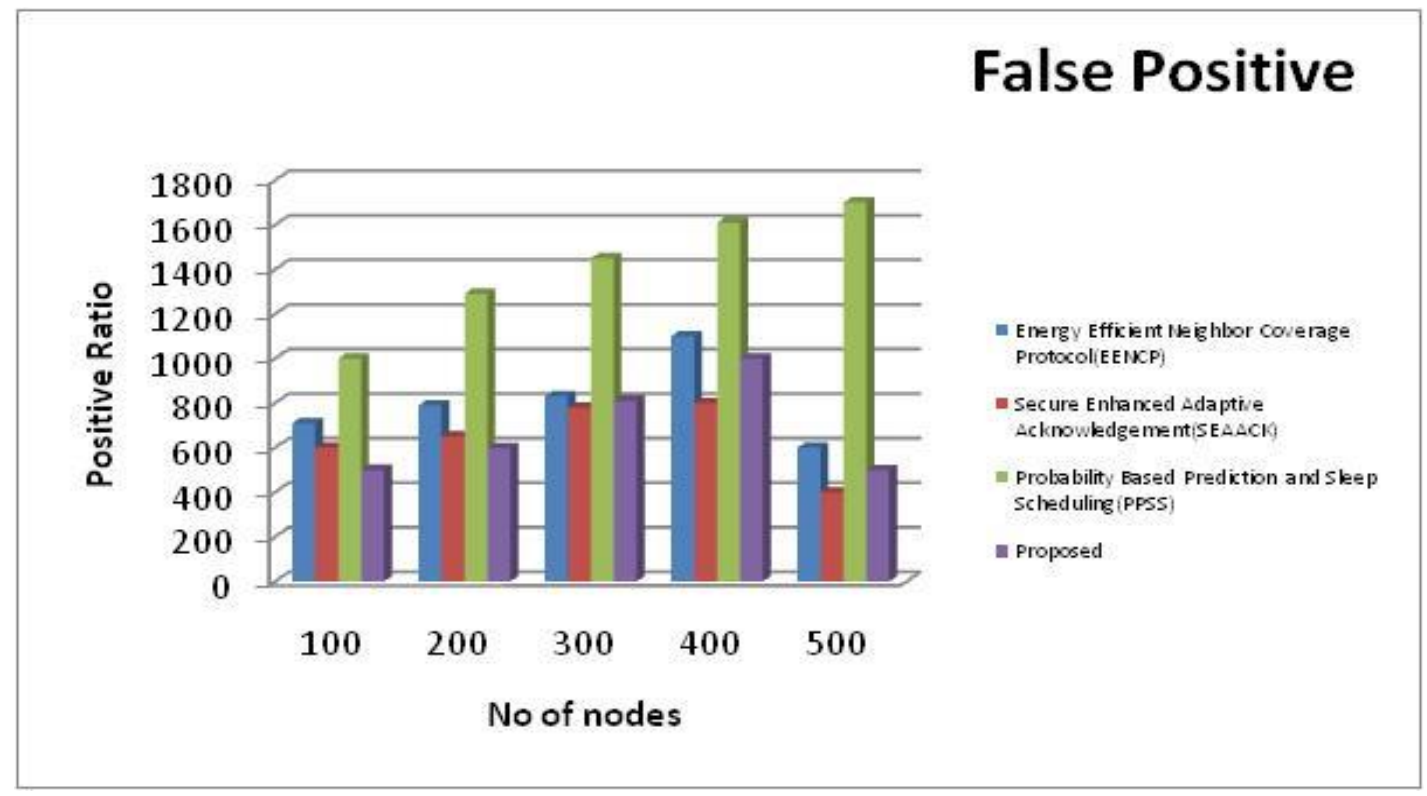

Figure 5: Comparison chart of false positive

The examination outline clarifies about the bogus positive proportion of three existing methods and one proposed method. The variations of its proportion is been clarified utilizing the positive proportion in $\mathrm{Y}$ hub and no of hubs in $\mathrm{X}$-hub. The chart shows the correlation of false positive proportion on the proposed and three existing methods. The bogus positive of proportion in proposed method is less in minimum 499 to greatest 1000 when contrasted with other existing methods.

\section{CONCLUSION}

The exhibition of area based intermediary methodology is contrasted and that of brought together and completely conveyed procedures. the information aggregation of LBAG is approved. Accumulated outcomes with semantic significance can be gotten. Moreover, we saw the extraordinary effect from fundamental MAC and directing layers on the exhibition of LBAG. With certain advancements on the GEOCAST steering layer convention, the general execution is improved incredibly. We at that point do sets of reproductions to test the conduct of three information aggregation methodologies: incorporated, completely circulated and Location Based Aggregation. Rather than executing conventions for unified and completely disseminated aggregation independently, we use LBAG to imitate their practices.

\section{REFERENCES}

1. Jeungeun Song, Yiming Miao, Enmin Song, M. ShamimHossain and Mohammed F. Alhamid, "Reliability-Aware Cooperative Node Sleeping and Clustering in Duty-Cycled Sensors Networks", Sensors 2018, 18, 127.

2. Hongbin Chen QianZeng and Feng Zhao, "Efficient Sleep Scheduling Algorithm for Target Tracking in Double-Storage Energy Harvesting Sensor Networks" , International Journal of Distributed Sensor Networks, 2016.

3. Jue Hong, Zhuo Li, Dianjie Lu and Sanglu Lu, "Sleeping Schedule-Aware Local Broadcast in Wireless Sensor Networks", International Journal of Distributed Sensor Networks, 2013.
4. Sha Liu, Kai-Wei Fan, PrasunSinha, "Dynamic Sleep Scheduling using Online Experimentation for Wireless Sensor Networks", 2014.

5. Niranjan Kumar Ray and Ashok Kumar Turuk, "A Hybrid Energy Efficient Protocol for Mobile Ad Hoc Networks", Journal of Computer Networks and Communications Volume 2016.

6. M. T. Hyland, B. E. Mullins, R. O. Baldwin, and M. A Temple, "Simulation-based performance evaluation of mobile ad hoc routing protocols in a swarm of unmanned aerial vehicles," in 21st International Conference on Advanced Information Networking and Applications Workshops (AINAW'07), vol. 02. IEEE Computer Society, 2007, pp. 249-256.

7. A. B. R. Kumar, L. C. Reddy, and P. S. Hiremath, "Performance comparison of wireless mobile ad-hoc network routing protocols," International Journal of Computer Science and Network Security (IJCSNS), vol. 8, pp. 337-343, 2008.

8. A. Neumann, C. Aichele, M. Lindner, and S Wunderlich, "Better Approach To Mobile Ad-hoc Networking (B.A.T.M.A.N.) draft-wunderlichopenmesh-manet-routing-00," Internet-Draft, Apr, 2008.

9. A. Koul, R. B. Patel, and V. K. Bhat, "Distance and frequency based route stability estimation in mobile adhoc networks," Journal of Emerging Technologies in Web Intelligence, vol. 2, no. 2, pp. 89-95, 2010.

10. J. Moy, "OSPF version 2," RFC 2328, April 1998.[Online]. http://tools.ietf.org/html/rfc2328

11. C. E. Perkins and P. Bhagwat, "Highly dynamic Destination-Sequenced Distance-Vector routing (DSDV) for mobile computers," in SIGCOMM Proceedings of the conference on Communications architectures, protocols and applications, vol. 24. London, England, UK: ACM, 1994, pp. 234-244.

12. T. Clausen and P. Jacquet, "Optimized Link State Routing Protocol (OLSR)," RFC 3626, Oct, 2003.

13. T. Clausen, C. Dearlove, P. Jacquet, and U. Herberg, "The Optimized198 BIBLIOGRAPHY Link State Routing Protocol version 2," Internet-Draft, March, 2012. 